unit of yeast or rat liver ribosomes was substituted for $E$. coli RNA.

It is, of course, impossible to say with certainty whether each single protein molecule has to re-enter its individual niche in the structure, or whether only a few key proteins of defined activitios must occupy their original positions. In any event, however, the spontaneous reassembly of a system of this complexity is as staggering as it is unprecedented. It offers exciting new possibilities for the study of ribosome function, and Traub and Nomura already give one example: they have found that the resistance to streptomycin of the ribosomes from streptomycin-resistant $E$. coli is associated not with the $16 S$ RNA but rather with the core proteins.

The progressive loss of proteins from $E$. coli ribosomes at increasing ionic strengths has been studied by Itoh, Otaka and Osawa (J. Mol. Biol., 33, 109; 1968). Whereas previously proteins had in general been removed in quite large batches, these workers have found that a more or less stepwise release can be achieved, using lithium chloride solutions under defined conditions. In the process the $50 \mathrm{~S}$ ribosomes are reported to give successively $40,36,28$ and $25 S$ products, and the $30 S$ ribosomes $25,23,21,19$ and $16 S$. The proteins remaining at each stage were identified by column chromatography, and their proportions determined by radioactive counting, the RNA and proteins being labelled respectively with tritium and ${ }^{14} \mathrm{C}$. They state that in the cores which remain after prolonged lithium chloride treatment, only one or perhaps two protein species remain. The results bear out earlier evidence of the dissociation of ribosomal proteins in several steps by a Russian group of workers.

\section{Location of RNA Cistrons}

\section{from our Cell Biology Correspondent}

The DNA sequences that specify $5 S, 23 S$ and $16 S$ ribosomal RNA and transfer RNAs in Bacillus subtilis are located in two clusters, one large and one small, in the bacterial chromosome. This is the picture that has emerged from some elegant experiments by Smith, Dubnau, Morell and Marmur (J. Mol. Biol., $33,123 ; 1968)$. In 1965 , these workers, and Oishi and Sueoka, independently, showed that the majority of the cistrons specifying $17 \mathrm{~S}$ and $23 \mathrm{~S}$ ribosomal RNA are located in a restricted portion of the $B$. subtilis genome. But these experiments gave no indication of the location of $5 S$ RNA, they did not resolve the order of the cistrons specifying each class of RNA and they did not show that all the RNA cistrons are in one cluster. The latest experiments clarify these questions.

To determine the order of replication of selected markers in the chromosome, Smith et al. took spores of $B$. subtilis grown on a light medium and germinated them in the presence of deuterium $\left(\mathrm{D}_{2} \mathrm{O}\right)$. In this system, newly replicated DNA will have a hybrid density and so can be separated from unreplicated DNA by density gradient centrifugation. By taking DNA samples at various times after germination and testing their ability to transform markers, the order of replication of those markers can be mapped. At the same time hybridization of the DNA with the various classes of RNA reveals the location of the RNA cistrons relative to the markers.
The result of these experiments indicates that between 60 and 80 per cent of the $5 S, 16 S$ and $23 S$ ribosomal RNA and transfer RNA cistrons lie in a cluster between the cistrons for streptomycin and puromycin resistance. The other 20 to 40 per cent of the RNA cistrons are probably clustered in a second region between cistrons involved in leucine and methione synthesis. The hybridization data indicate that there are four $5 S$, nine to ten $16 S$ and $23 S$ and about forty transfer RNA cistrons in the entire genome. Thus the larger cluster must include two or three $5 \mathrm{~S}$ and six to eight $16 S$ and $23 S$ ribosomal RNA cistrons and about thirty transfer RNA cistrons. In this cluster the average map position of the RNA cistrons is strep, $23 S, 16 S$, transfer, puro, and the $5 S$ RNA cistrons are close to the $23 S$ and $16 S$ RNA loci.

What is the significance of the clustering of the ribosomal RNA cistrons? The obvious suggestion, made by Dubnau, Smith and Marmur in 1965, is that it reflects the existence of some co-ordinated control mechanism for the synthesis of the ribosomal RNAs. Certainly the discovery that $5 S$ RNA cistrons are localized with the $16 S$ and $23 S$ cistrons supports this view. The close linkage between ribosomal RNA cistrons and cistrons conferring resistance to antibiotics including streptomycin, puromycin, erythromycin and kanamycin is also consistent. It is known that several of the cistrons that confer resistance to these antibiotics are probably concerned directly with the synthesis of the $30 S$ ribosomal sub-unit.

It is also significant that Cutler and Evans (see Nature, 215, 7; 1967) found two distinct regions in the E. coli chromosome which are complementary to $16 \mathrm{~S}$ and $23 S$ ribosomal RNA and transfer RNA. Moreover, these regions contain the cistron for streptomycin resistance. As Smith et al. suggest, mutation to streptomycin resistance may occur in a $16 \mathrm{~S}$ ribosomal RNA cistron.

\section{Mitochondria producing Heat}

\section{from our Medical Biochemistry Correspondent}

Sомє newborn mammals, hibernating mammals and mammals which have been acclimatized to cold conditions often contain large amounts of brown adipose tissue. This is important in maintaining body temperature because it is a very efficient heat producer as well as acting as an insulator, like white fat. Each cell in brown fat contains a very large number of mitochondria, which are presumed to release energy as heat during their respiration. Numerous workers have found that the $\mathrm{P}$ : $\mathrm{O}$ ratio (molecules of adenosine triphosphate (ATP) formed for each molecule of oxygen consumed) of mitochondria isolated from brown fat was very low, so it was thought that electron transport in these mitochondria was permanently uncoupled from the reactions which normally produce ATP.

Two groups of workers have, however, recently shown that the mitochondria from brown fat can in fact produce as much ATP as other mitochondria when they are incubated in the presence of defatted serum albumin. Both groups isolated mitochondria from the brown fat of newborn rabbits and rats which had been acclimatized to cold for several weeks. Guillory and Racker (Biochim. Biophys. Acta, 153, 490; 1968) measured the $\mathrm{P}: \mathrm{O}$ ratios of the mitochondria using a-ketoglutarate and succinate as substrates, while 